\title{
Effect of Y-Length and Biasing of Lithium Niobate Mach-Zehnder Modulator on Insertion Loss and Extinction Ratio
}

\author{
Nor Hidayah Roslan, Aziati Husna Awang \& Mohd Hanapiah M. Yusoff
}

\begin{abstract}
An optical modulator is a device which signalcontrolled the electro-optic effect which is used to modulate the beam of light of an optical signal. This article analyzes the performance of different structures of Mach-Zehnder Interferometer (MZI) modulator. The conventional MZI modulator high loss compare to the PCW modulator. So that we compares the performance of the photonic crystal waveguide (PCW) and conventional of Mach-Zehnder Interferometer (MZI) modulator on the lithium niobate (LinBO $)_{3}$ ).(Method)For the PCW of MZI modulator is designed photonic crystal cavity at one arm of the modulator then for the conventional MZI modulator there is a electrode at one arm of the modulator and applied voltage of $0.9 \mathrm{~V}$. This project used OptiFDTD, OptiBPM software for designing the structures of both modulators and performance analysis. The performance has been analyzed based on the insertion loss, extinction ratio and the phase difference. Low insertion loss with increasing the length of $Y$ branch for the PCW of MZI modulator compare to the conventional MZI modulator. It has highest extinction ratio $8.0521 \mathrm{~dB}$ compare to the conventional MZI modulator only $1.29 \mathrm{~dB}$ and the phase difference is bigger for the PCW of MZI modulator compare to the conventional MZI modulator to achieve the low power consumption for the optical interconnects in computer chip performance.Therefore, PCW of MZI modulator has low insertion loss and high extinction ratio to obtain good value of modulation efficiency and high phase shift to achieve better performance.
\end{abstract}

Keywords-Optical modulator, MZI, PCW, $\mathrm{LiNbO}_{3}$, OptiFDTD, OptiBPM.

\section{INTRODUCTION}

The primary purpose of an optical modulator is to modulate the optical signal by varying the characteristics of the light beam such as amplitude, phase or polarization [1].

This manuscript is submitted on 3 April 2019 and accepted on 24 November 2019. Nor Hidayah Roslan and Aziati Husna are with the Faculty of Electrical Engineering, Universiti Teknologi MARA, 40450 Shah Alam, Selangor (e-mail: noorhidayah108@gmail.com)

Mohd Hanapiah M. Yusoff is with Faculty of Applied Sciences,Universiti Teknologi MARA, 40450 Shah Alam, Selangor
The optical signal from the light beam in a waveguide can be possible with the applied electric field would change in the real or imaginary parts of refractive index of the material used, which is known as electro-refraction and electro-absorption, respectively in optical modulator [2]. The primary electric field effects that are traditionally useful in semiconductor materials for causing either electro-absorption or electrorefraction are the Pockels effect and the Kerr effect.Pockels effect changes or produces birefringence in an optical medium induced by an electric field and Kerr effect change of refractive index of materials in response to a applied electric field. The Kerr effect is distinct from the Pockels effect in that the induced index change is directly proportional to the square of the electric field instead of varying linearly with it. Furthermore there are optical behaviors for the photonic crystal waveguide (PCW) has provided [3]. In conventional photonic crystal systems, extrinsic scattering resulting from random manufacturing defects or environmental changes is a major source of loss that causes performance degradation, and the backscattering loss is amplified as the group velocity slows down. In order to overcome the limitations in slow light systems, we propose a backscattering-immune slow light waveguide design such as photonic crystal waveguide (PCW).

The material properties of Lithium Niobate (LN) are ultrafast modulation, low voltage operation and also low optical losses at the same time [4].Furthermore the microstructured LN devices can provide better modulator performance most commercial LN modulators are still based on titanium-in diffusion or proton-exchange waveguides, because $\mathrm{LN}$ is notoriously difficult to etch. These waveguides typically have a low refractive index contrast $\Delta \mathrm{n}$ of around 0.02 between core and cladding, resulting in a large optical mode size. The weak optical confinement requires metal electrodes to be spaced far apart from the optical waveguide (about $10 \mu \mathrm{m}$ ), lowering the electro-optic efficiency. As a result, LN modulators today are much larger in size and require much higher drive voltages than the material is capable of supporting.. (Discuss and highlight research gap). The previous research focused on silicon as the substrate, that has the advantage of maintaining maximum compatibility with the existing CMOS fabrication infrastructure. However, it is not the best possible choice because of its passive optical behaviour due to weak electro-optic response that complicates 
the design of compact and high-performance of the optical devices [5]. Therefore, we have chosen the $\mathrm{LN}$ as an active material to implement photonic crystal slow light structures that leads to large electro-optic coefficient and fast response of time [6].

The potential application of photonic crystal modulator that implements the slow light can further improved matter interactions, has been research focus in the field of an optical communications [7]. The slow light technique can enhance the nonlinear effect in the photonic crystal structure and also reducing the $\pi$ - voltage of electro-optic modulator thus can reduce power consumption. In this paper, we reported the investigation the MZI modulator with electrodes in one arm known as the phase modulator [8-9].The phase modulator changes the the refractive index material and the phase optical signal that passes through it also changed [10]. Using the OptiBPM software, we show the effect length structure of the electrode on the performance of the conventional modulator in order to reduce the insertion loss and the phase shift occurred and for the photonic crystal waveguide modulator is designed using FDTD OpticWave software where the slow light technique is implemented.

The design which includes the both of the design photonic crystal and conventional MZI modulator. The methodology was applied to obtain the best performance that can reduce the insertion loss and the phase shift will change. The insertion loss is one of the most important factors in the optical power loss when the modulator is coupled to a photonic circuit. This factor affects the loss of the budget and overall connection system [11]. The phase modulator which change the 'phase' of optical signal that passes through the modulator by applying voltage. When voltage is not applied to the RF-electrode, $n$ numbers of waves exist in the certain length. When voltage is applied to the RF-electrode, one more wave is added, which now means $\mathrm{n}+1$ waves exist in the same length. In this case, the phase has been changed by $2 \pi$ (360degree) and the half voltage of this is called the driving voltage. The phase modulator is applied to compensate for this degradation due to non-linear effect such as self-phase modulation; it is possible to transmit long distance optical transmission [12].

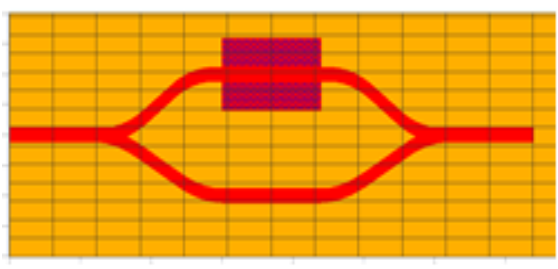

(a)

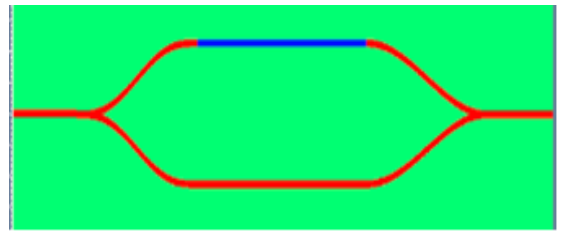

(b)

Fig. 1: (a)The photonic crystal waveguide (PCW) and (b)conventional MZI modulators

\section{OPTICAL MODULATOR DESIGN STRUCTURES}

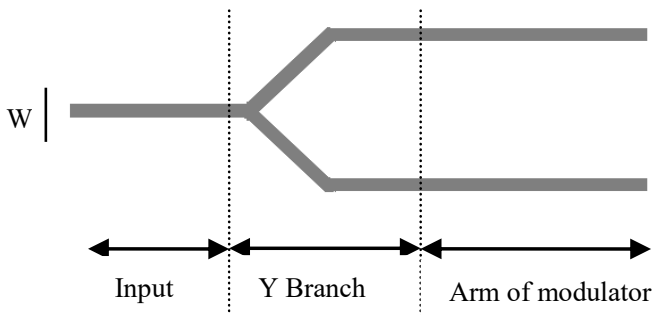

(a)

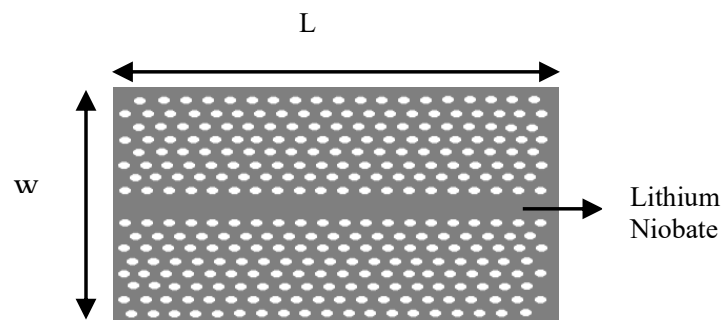

(b)

Fig. 2: The structures of $\mathrm{Y}$-junction and photonic crystal waveguide (PCW) of MZI modulator.

Figure 2 above shows the structure of the MZI modulator and the photonic crystal cavity. The width of the modulator is $\mathrm{W}=$ $1.5 \mu \mathrm{m}$ with the thickness $220 \mathrm{~nm}$ that support a single-mode same as the thickness of PCW. The input waveguide is 15 $\mu \mathrm{m}$,the length of $\mathrm{Y}$ branch is $22.5 \mu \mathrm{m}$ and the length arm of modulator is $17.5 \mu \mathrm{m}$ the other one arm acts as an optical phase shifter. For the 2D hexagonal photonic crystal cavity (PCW), the width is $\mathrm{W}=7.5 \mu \mathrm{m}$ and the length, $\mathrm{L}=17.5 \mu \mathrm{m}$. All the radius of holes are same where $\mathrm{R}=0.1075 \mu \mathrm{m}$ and the chosen of lattice constant $\mathrm{a}=0.4 \mu \mathrm{m}$ in order to reduce the losses. This design operate at communication wavelength $\lambda=$ $1.3 \mu \mathrm{m}$. (Selection parameters need to justified) Moreover, a single line defect waveguide is created by removing the full row of cylinders, that leads to photonic band gaps (PBGs) where the electromagnetic modes cannot propagate inside the structure [13]. Both materials for the MZI modulator and PCW are using lithium niobate. Lithium niobate (LN) as active material to realize photonic crystal slow light structures because of its large electro-optic coefficient and fast response time [14].

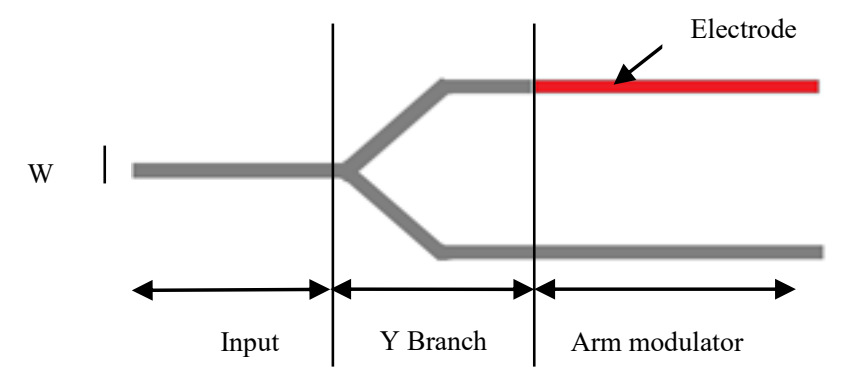

Fig. 3: The structure of conventional MZI modulator 
The structure of conventional MZI modulator shown above in Figure 3. The width of MZI modulator, $\mathrm{W}=5 \mu \mathrm{m}$, the length of input is $750 \mu \mathrm{m}$ and $\mathrm{Y}$ branch is $800 \mu \mathrm{m}$. Both length of arm modulator and electrode are in the same length $3150 \mu \mathrm{m}$. The thickness is $2 \mu \mathrm{m}$ and placed the gold planar waveguide electrode as shown in figure above. The refractive index for the gold electrode $n=0.27732$. The electrode is grounded at one arm of the modulator and a modulating voltage $0.9 \mathrm{~V}$ that generate a volatge wave through the waveguide so that the electric modulation field along the $\mathrm{Z}$ - direction.

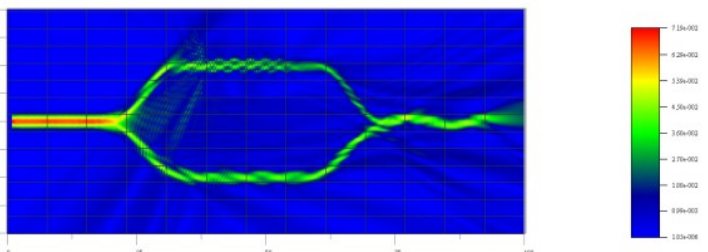

Fig. 4: PCW with MZI modulator optical field in FDTD

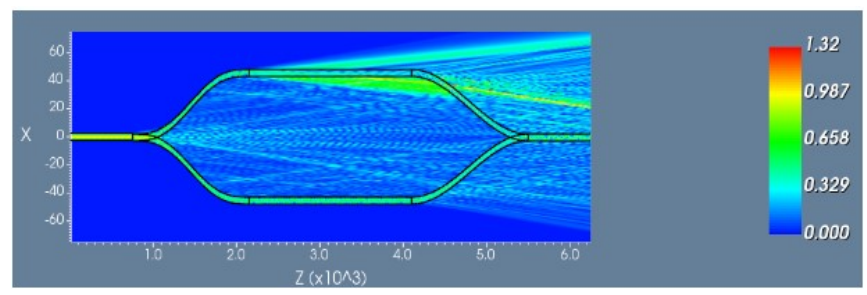

Fig. 5: Conventional MZI modulator optical field in OptiBPM

The design of the PCW and conventional MZI modulator were done in FDTD and OptiBPM. Figure 4 and Figure 5 show the light intensity flow through the waveguide of MZI modulator. At PCW with MZI modulator there is loss at one arm of the modulator due to the reflection and light scattering, also same goes to the conventional MZI modulator.

\section{RESULTS AND DISCUSSION}

In Figure 6 above shows the insertion loss of the photonic crystal waveguide of MZI modulator where the result show that the insertion loss increasing with the increasing the length of Y Branch. This is leads to the light from the source to the reflection and scattering light compared to the shortest length of $\mathrm{Y}$ branch so that the insertion loss is small $-34.6139 \mathrm{~dB}$. While the insertion loss for the conventional MZI modulator is also the same with the PCW MZI modulator where the insertion loss also low when the length of $\mathrm{Y}$ branch is shorter. From the result at Figure 7 we can see that the lowest insertion loss $-10.7 \mathrm{~dB}$. The insertion loss has been calculated by using this formula IL $(\mathrm{dB})=\mathrm{PO}-\mathrm{Pi}$, the difference of the output power and the input power of the both PCW MZI modulator and conventional MZI modulator. The performance of conventional MZI modulator of the insertion loss can be observed through the optical power meter in OptiSystem. The insertion loss is a measure of loss across a circuit due to all factors, including absorption, bending loss from macrobends and microbends, diffusion, dispersion, Fresnel reflection, and leaky modes.

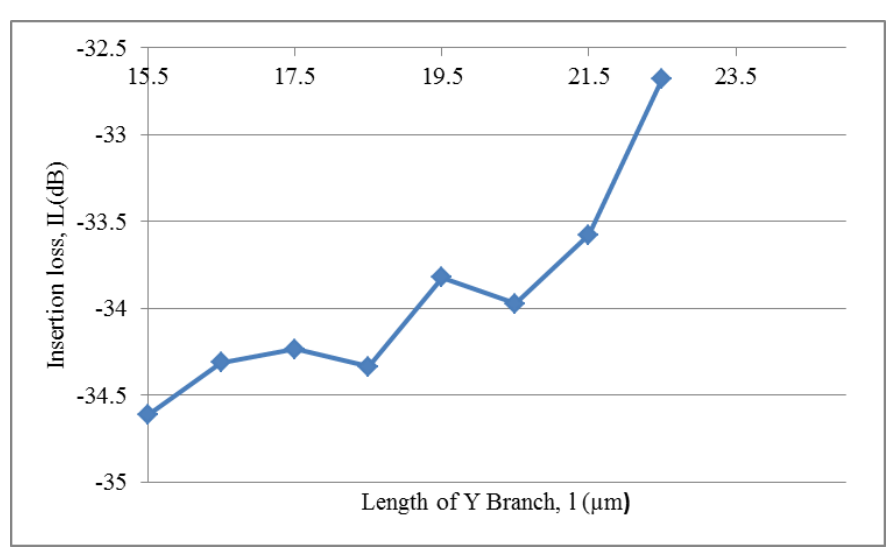

Fig. 6: The insertion loss of PCW MZI modulator

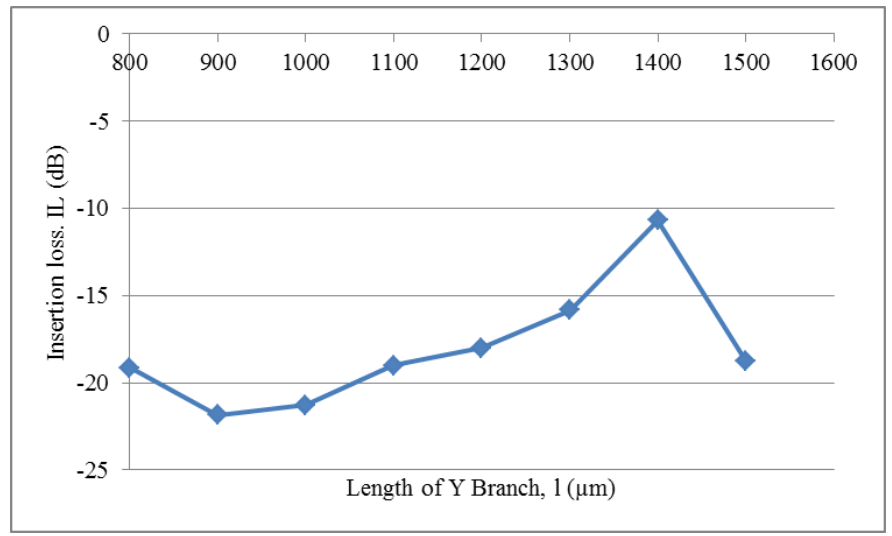

Fig. 7: The insertion loss of conventional MZI modulator

To further clarify the effect of different between PCW MZI modulator and conventional MZI modulator structure, the extinction ratio has been studied. Low insertion loss is good value of the modulation efficiency and low loss of the signal power resulting from the insertion of the device. The extinction ratio (re) is the ratio of two optical power levels of a digital signal generated by an optical source. The extinction ratio may be expressed as a fraction, in $\mathrm{dB}$, or as a percentage. It is been calculated by using this formula $\mathrm{re}=\mathrm{Pi} / \mathrm{PO}$, where $\mathrm{Pi}$ is optical power level generated when the light source is on and $\mathrm{Po}$ is the power level generated when light source is off. Refer to the Figure 8 above, the extinction ratio decreasing when the length of $Y$ branch increased. The highest extinction ratio is $8.0521 \mathrm{~dB}$ at length of $\mathrm{Y}$ branch, $\mathrm{l}=15.5 \mu \mathrm{m}$. It is different with the extinction ratio for the conventional MZI modulator. From the Figure 9 above shows that the highest extinction ratio which is $1.29 \mathrm{~dB}$ at length of the $\mathrm{Y}$ branch, $\mathrm{l}=$ $1400 \mu \mathrm{m}$. In general, the requirement of extinction ratio is over $20 \mathrm{~dB}$ for the commercial product specification. To meet the condition, both structures of PCW and conventional MZI modulator need improvement but we choose the PCW MZI modulator with the highest extinction ratio compare the structure of conventional MZI modulator. As the extinction ratio improves, the bit-error ratio (BER) improves, reducing the number of errors and the amount of error correction required. Briefly, poorer values of ER increase the power 
penalty (PP), worsen BER, and diminish the benefit of increased power.

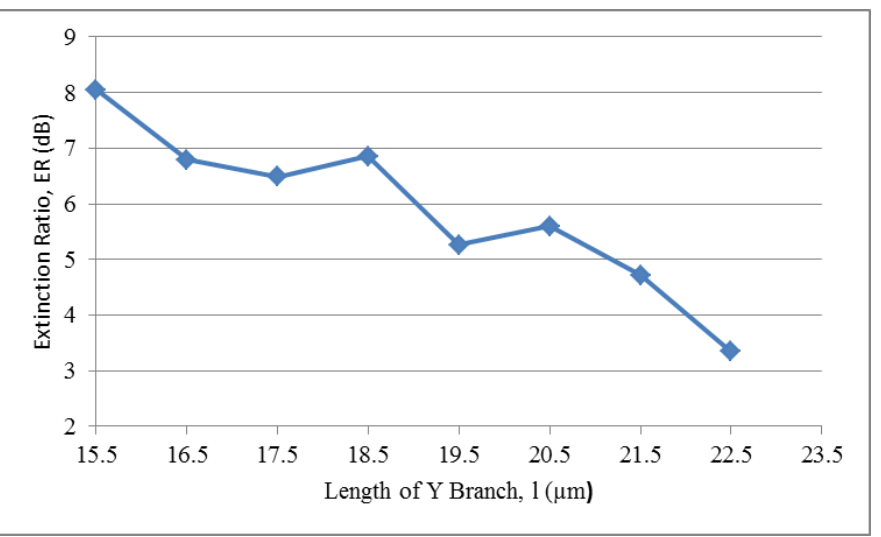

Fig. 8: The extinction ratio of PCW MZI modulator

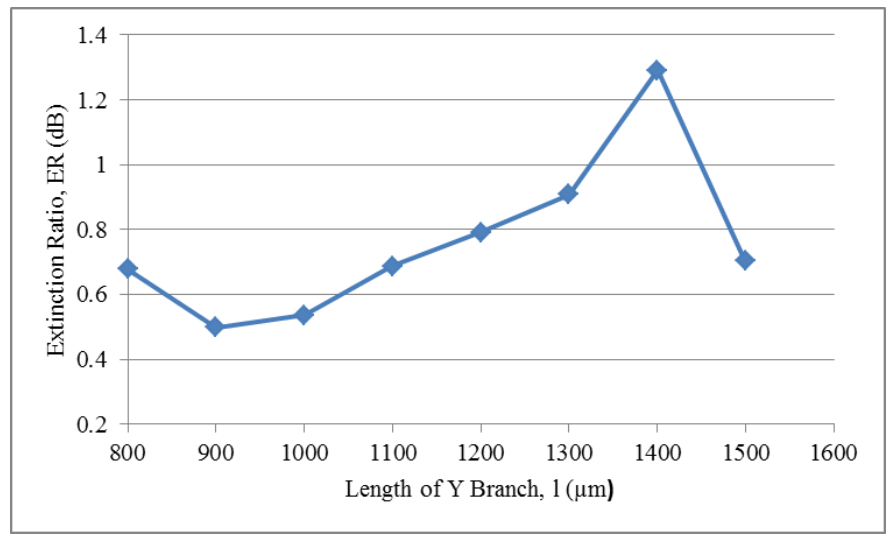

Fig. 9: The extinction ratio of conventional MZI modulator

The other important performance of the MZI modulator sensitivity is characterized by the $\pi$-Voltage $U_{\pi}$ of its phase difference. Phase Difference $(\phi)$ between two particles or two waves tells us how much a particle (or wave) is in front or behind another particle (or wave). Here some calculation to find the phase difference path length at one active arm of the modulator should be done. The phase difference/ phase shift $\Delta \Phi$ for the photonic crystal waveguide and conventional is given by the formula $\Delta \phi=\phi 1(\lambda)-\phi 2(\lambda)=2 \pi \mathrm{L}((\mathrm{n} 1(\lambda)-$ $\mathrm{n} 2(\lambda) / \lambda)$, where $\mathrm{L}$ is the length of the active arm, for the conventional MZI modulator the $\mathrm{L}$ is the length of the electrode. The effective refractive index $n 1$ is for the upper arm or the active arm and the $\mathrm{n} 2$ is the refractive index for the lower arm of the modulator. For PCW, the effective refractive index is calculated $n_{\text {eff }}=\beta / k_{\mathrm{o}}$ where $\beta$ is the propagation constant and $\mathrm{k}_{\mathrm{o}}$ is the wave vector. The effective refractive index for $\mathrm{LN}$ is $\mathrm{n}_{\mathrm{eff}}=2.2$. Then $\lambda$ is the range of the wavelength. From the both figures above Figure 10 and Figure 11 show that the highest phase difference when the active arms are longer. The highest phase difference for the PCW with MZI modulator is $-3.9345(\mathrm{rad} / \pi)$ at length of the active arm, $\mathrm{L}=15.5 \mu \mathrm{m}$. We achieve this length reduction by using PC line defect waveguide that is designed to have low optical group velocity and thus can increased electro-optic interaction. while for the conventional MZI modulator the highest phase difference is $-6.35(\mathrm{rad} / \pi)$ where the length of active arm at $\mathrm{L}=$ $1750 \mu \mathrm{m}$. The highest phase difference gives a better performance of the device. Furthermore The phase, $\phi$, of the light wave is directly proportional to the optical path length traveled by the light, which is controlled by $V(t)$. The applied driving voltage, $V(t)$, is often expressed in terms of the halfwave driving voltage, $V \pi$. Driving an EO phase modulator at its half-wave voltage generates a phase shift equal to pi: $\phi(t)$ $=\pi$. The modulation voltage $U$ that is required to change the phase in one modulator arm by $\pi$ is called $\pi$-voltage $U_{\pi}$ The voltage applied to the electrode is $0.9 \mathrm{~V}$ for all the different length of active arm, but the phase difference also shifted at different wavelength. In PCW waveguide MZI modulator the incident light from the light source is split into two waveguides. The output amplitude depends on the phase difference at recombination. In active arm recombination gives a ' 1 ' bit output while the passive arm recombination gives a ' 0 ' bit output. Moreover, if the waveguide in MZI modulator is placed with the photonic crystal cavity waveguide, the effect of a material nonlinearity can be enhanced instead of low power implement the slow light technique on the MZI modulator. The cavity of the photonic crystal that controlled the light through the waveguide so that the signal flow will be delay and the phase difference occurred to the MZI modulator. Designed PCW usually support a mode low group velocity and flat dispersion curve and implement the slow light of the PCW of MZI modulator. Hence, the one of the modulator arm is delay the phase difference that shifts is not same with the phase of the other arm of that modulator so that the power maybe can be reduced.

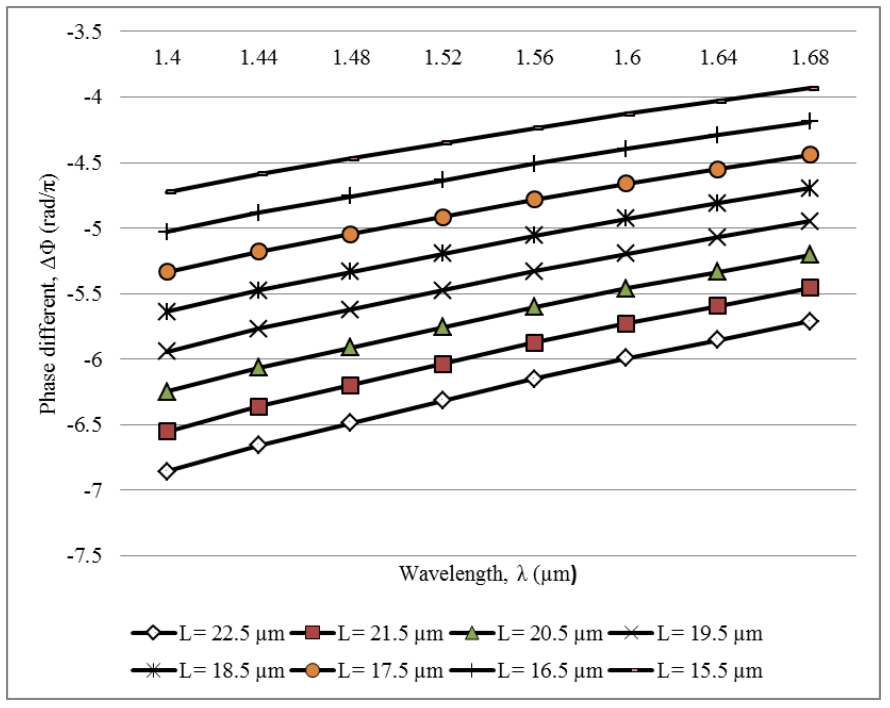

Fig. 10: The phase difference of PCW MZI modulator 


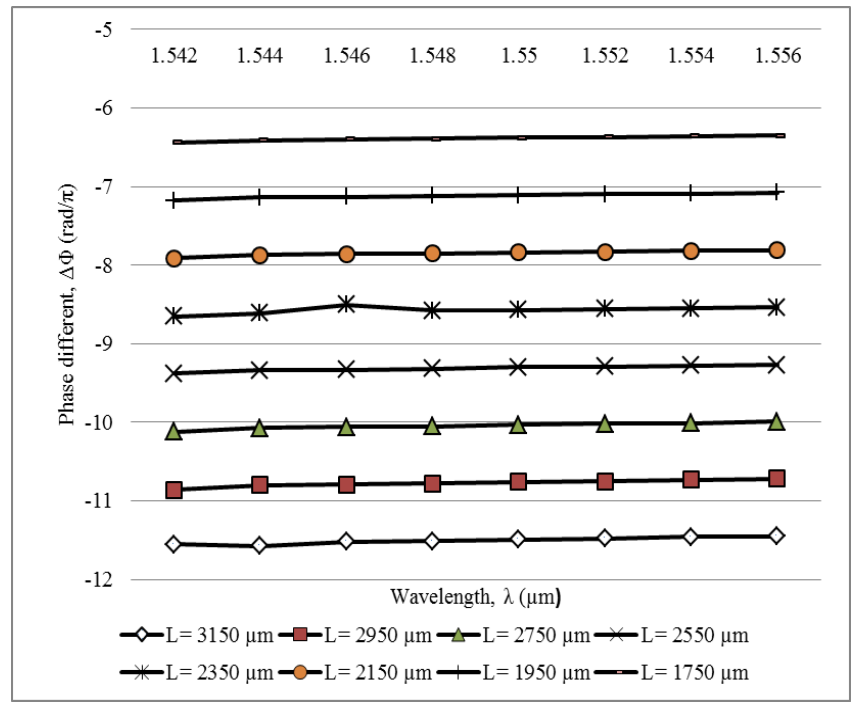

Fig. 11: The phase different of conventional MZI modulator

\section{CONCLUSION}

In conclusion, the PCW of MZI modulator structure and conventional MZI modulator are investigated by using the FDTD and OptiBPM software. The PCW of MZI modulator implement the slow light technique shows that low insertion loss $34.6139 \mathrm{~dB}$ at $15.5 \mu \mathrm{m}$ of the length $\mathrm{Y}$ branch. It has highest extinction ratio $8.0521 \mathrm{~dB}$ compare to the conventional MZI modulator only $1.29 \mathrm{~dB}$ and the phase difference for the photonic crystal waveguide which is implement the slow light technique can enhance the material nonlinearity and can achieve the low power consumption.

\section{ACKNOWLEDGMENT}

The authors thank the Faculty of Electrical Engineering and Institute of Research Management and Innovation (IRMI) of UiTM for their support.

\section{REFERENCES}

[1] A. Govdeli, M. C. Sarihan, U. Karaca, and S. Kocaman, "Integrated Optical Modulator Based on Transition between Photonic Bands," Sci. Rep., vol. 8, no. 1, pp. 1-11, 2018.

[2] G. T. Reed, G. Mashanovich, F. Y. Gardes, and D. J. Thomson, "Silicon optical modulators," Nat. Photonics, vol. 4, no. 8, pp. 518$526,2010$.

[3] Cai, W. Optical metamaterials: fundamentals and applications. (Springer, 2016).

[4] C. Wang et al., "Integrated lithium niobate electro-optic modulators operating at CMOS-compatible voltages," Nature, vol. 562, no. 7725, pp. 101-104, 2018.

[5] X. Wang, H. Tian, and Y. Ji, "Photonic crystal slow light MachZehnder interferometer modulator for optical interconnects," $J$. Opt., vol. 12, no. 6, 2010.

[6] J. D. Witmer, J. A. Valery, P. Arrangoiz-Arriola, C. J. Sarabalis, J. T. Hill, and A. H. Safavi-Naeini, "High-Q photonic resonators and electro-optic coupling using silicon-on-lithium-niobate," Sci. Rep., vol. 7, pp. 1-7, 2017.

[7] S. Serna et al., "Experimental GVD engineering in slow light slot photonic crystal waveguides," Sci. Rep., vol. 6, pp. 1-9, 2016.

[8] K. Debnath et al., "All-silicon carrier accumulation modulator based on a lateral metal-oxide-semiconductor capacitor," Photonics Res., vol. 6 , no. 5 , p. 373,2018
[9] R. W. Purnamaningsih, N. R. Poespawati, I. Saraswati, and E. Dogheche, "Design of GaN based optical modulator with machzehnder interferometer structure," WSEAS Trans. Commun., vol. 13, pp. 229-233, 2014.

[10] L. P. Laurent Viviem, Silicon Photonics Hnadbook of $1^{\text {st }}$ ed. CRC Press, 2013.

[11] H. Hazura, S. K. Idris, A. S. M. Zain, F. Salehuddin, and P. S. Menon, "Modeling Mach Zehnder Interferometer ( MZI ) Modulator on Silicon-On-Insulator ( SOI )," J. Telecomunication Electron. Comput. Eng., vol. 8, no. 1, pp. 121-124, 2016.

[12] SUMITOMO OSAKA CEMENT CO., "Application Note for LN Modulators," Control, 2007.

[13] S. Rao, R. Kisacik, T. Tekin, M. Casalino, G. Coppola and F. G. Della Corte, "Use of amarphous silicon for the design of a photonic crystal based MZ modulator at $1.55 \mu \mathrm{m}, " 2015$ Fotonica AEIT Italian Conference on Photonics Technologies, Nov 2015. 